\title{
Comparison of different algorithms to improve the quality of Image
}

\author{
Mr. Mayur M. Sonavane, Dr. S. S. Agrawal \\ M.E. Student of E\&TC, SKNCOE Pune, India \\ Assistant professor of E\&TC SKNCOE Pune, India
}

\begin{abstract}
Super-resolution (SR) become the most important task in image processing because of requirement of high quality of images. It becomes popular in technology like computer vision and pattern recognition. In Single Image Super-resolution (SR) uses the sequences of single low resolution (LR) images from high resolution (HR) to improve the quality of image. The $S R$ is an image processing problem which aims to generate a high resolution (HR) image from low resolution input image. The SR is difficult because of the missing information in the given LR image. Thus in SR it is very important to boost the reconstruction performance so that, there is need of effective prior knowledge. The prior knowledge of image will solve the problem to improve the quality of generated image. The main aim of SR is to generate the HR image with good visual perception as similar as original image. The paper compare two methods of SR that is bi-linear method, and direction group sparsity of image method that is proposed one. These methods will evaluate the accuracy, subjective visual effect, quality of image, time complexity and PSNR, etc. These calculations will enhance nature of picture like better visual impact quality, lower reconstruction error and adequate calculation proficiency, and so on.
\end{abstract}

Keywords: Accuracy, bi-linear SR, Directional group sparsity, PSNR, Super-Resolution.

\section{Introduction}

The high resolution imaging systems, gives better performance in image analysis and pattern recognition so that Super-resolution technique is introduced. By using high quality sensor in the camera, it is easy to capture the high resolved image but this becomes more expensive and costly. In the Super- resolution technique the high quality sensors system in camera is replaced by image processing algorithms, which is relatively less expensive to implement. The use of such algorithm will be continue, where the low quality sensors are used and high-quality imaging systems necessary. The basic technique behind Super-Resolution is fuse the sequence of low-resolution images and will result a high resolution image. One of the main problem in Super-Resolution is aliasing effect and this effect can be completely removed if a relative sub-pixel movement is exists between the under examined input images. The reason behind to make Super-Resolution one of the most popular research area of image processing is it's computationally complexity and numerically ill-posed problem.

The sensors used in the camera or the image acquisition device is main limitation of image resolution. The complementary metal-oxide-semiconductor (CMOS) or a charge-coupled device (CCD) these two are used as image sensors. To capture the two dimensional images sensors are also arranged in two dimensional array. The size of sensor used or number of sensors element used per unit area decides the quality of image. As increase in the density of sensor elements also increases the quality of image but this is most costly. If decrease in number of sensor elements per unit area also decreases the quality of captured image. The spatial resolution of the image, is limited by the image sensors. The image quality are also degraded by the optics, due to lens blurs, lens aberration effects, aperture directions and optical blurring due to motion. The quality of image will be improve by using the sequence of degraded images and then fuse all these sequences using image processing algorithm. This is main aim of this paper, which is without changing hardware of device improve the quality of image by using signal processing algorithm.

\section{Literature Survey}

There are various techniques are used for Super-resolution of Image. The Super-resolution is mainly used to construct the high resolution image from the series of low resolution images. The image registration and image reconstruction are two main processes of Super-Resolution. Image super-resolution reconstruction is rapidly growing research area. The reconstruction algorithms are divided into three categories.

1. Interpolation techniques [2], [3] is most commonly used in Super-Resolution of image. They gauge the missing pixels in the high resolved image with their known neighbors to overhaul the input low resolved image. The complexity of these methods is not good. They produces the error like blurring effects along the edges. The advantage of interpolation based method is they take low computational load and therefore real time application is possible. This method is not useful for high quality Super-resolution reconstruction. 
2. In Learning based strategies [4], [5] the mapping between low resolution and high resolution image patch from outside preparing dataset is evaluated for high resolution image. The soft decision method estimates the missing pixel blocks by adaptively learning spatial sample relationship as well as between known and unknown pixels.

3. Reconstruction techniques generally depends previous knowledge of the Super-resolved image. Then they estimate the reconstruction constraints by degrading the high resolved image to low resolved image. The system in urban scene or synthetic environment considering their 3D geometry. They have exceedingly organized straight edges. The previous knowledge is extracted by model of first order conic solvers (TFOCS) [6], transform invariant (TI) and directional total variation (DTV) regularizations [7], [8]. This permits to acquire a novel curved regularizer that essential global consistency restrictions between the edges of the image. Suppose the pixel of priory knowledge is located near the edge then this pixel will have stronger influence in filtering. In such case steering kernel regression (SKK) [9] is used to measure the function of local gradient between the neighboring pixels to estimate the weight. Pixels to estimate the weight.

The main objective of Super-resolution is

1. The image registration and reconstruction is a fundamental image processing problem that is well known illposed. The reconstruction of image should be same as original image that is the main aim.

2. Computational efficiency is another main objective or Super-resolution of image due to large number of unknowns requires more number of matrix calculation.

3. The algorithm should give better visual quality of image.

4. Robustness of Super-resolution is of the most important parameter because the image degradation model parameters cannot be estimated perfectly.

5. Traditional Super-resolution techniques are vulnerable because of error in motion, less accurate blur models, moving objects, blur due to motion, etc. The quality of image should be improved because of these effects.

\subsection{Mathematical Model:}

\section{Methodology}

In mathematical model, we had constructed the matrix model of $M$. There are basically three main parameters to explore the image formation model that is motion into image, sampling process and blurring. These three terms can be modeled as mathematically in matrix form as

$$
M=D A H F
$$

Where $\mathrm{F}$ is the intensity conserving, geometric wrap operation catching picture movement, $\mathrm{H}$ is the obscuring operation because of the optical point spread capacity (PSF) and D and A is the impact of inspecting by the picture sensor.

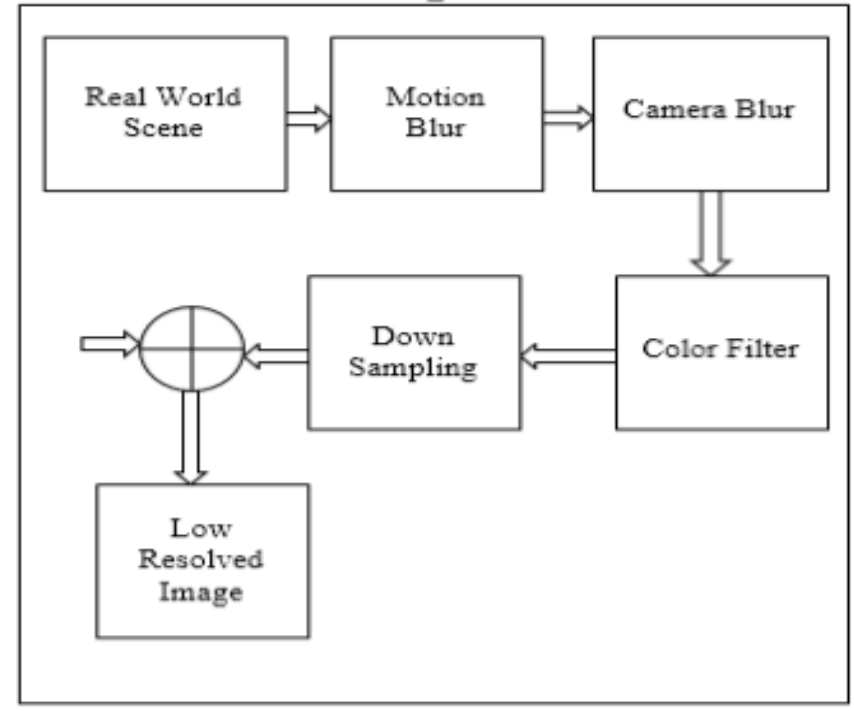

Fig. 1 Degradation Model of Image

At each stage these modeling terms would catch the genuine impacts of the picture arrangement process. By and by, be that as it may, the models utilized mirror a mix of computational and measurable constraints. This permits to use steady and productive calculations for evaluating blurring function Or, the resolution upgrade element relies on upon the quantity of low resolution outline, the computational confinements and the exactness of motion estimate. Because of off base estimate there may critical diminishment in general execution. 


\subsection{Proposed Method}

This paper proposed the reconstruction method which calculate the sparsely placed directional features of image and gradient of direction. The edges of images directed in very less direction. Due to artificial blur in sharp edges the reconstruction quality is affected.

By taking the average of neighbor pixels we can eliminate the artificial blur between the sharp edges. Use pixel value of local image patch and directional features of center position to estimate the weight. To separate the primary course of sharp edges of high resolved image use utilize the directional gathering sparsity in image which gives the SR quality. On the off chance that we utilize just the pixel values in the neighbor look this lead to low coordinating. So we use both the pixel values and the directional components to process the similarity weights.

Iteratively minimizing the remaking blunder until the most extreme number of cycles is come to. In outline, there are three fundamental commitments of the proposed technique:

1. The group sparsity property of image gradients is utilized to locate the key headings. A CTV term is adequately protect the sharp edges in 16 unique headings.

2. A D-NLM term is proposed to successfully choose those neighbors with comparable pixels and in addition comparative directional data.

3. A SR system joins the reconstruction imperative, D-NLM and CTV terms. An ideal answer for this SR recreation issue is acquired by using the TFOCS system.

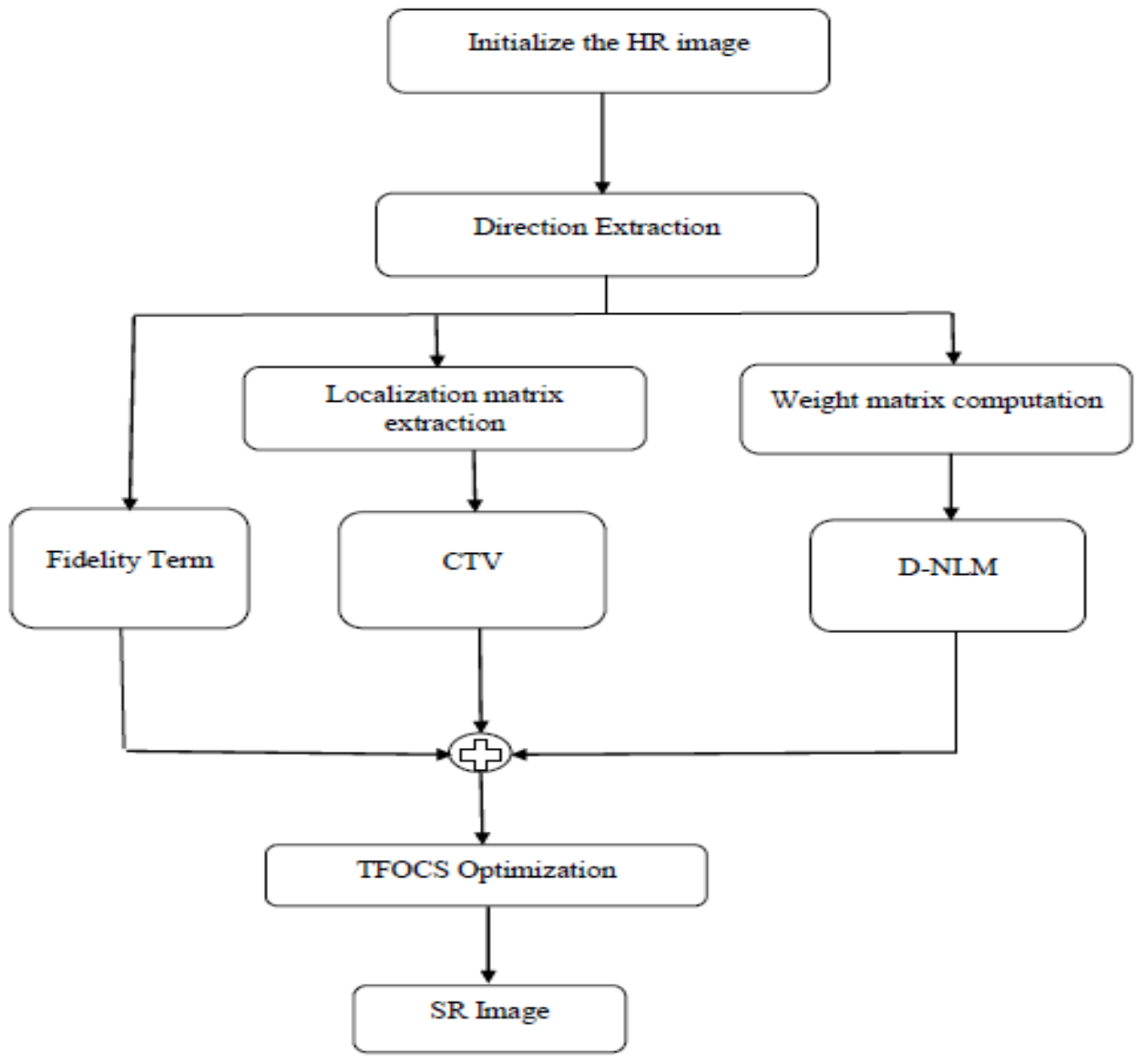

Fig. 2 Flow Chart of proposed method

The issue of super-resolution a picture got from normal scenes is curio between sharp edges can be suppress by utilizing, propose a novel reproduction based SR system which utilize two earlier terms CTV and DNLM and the global reconstruction imperative to guarantee that the SR reconstruction can be very much postured. To successfully separate the directional data of a characteristic image, by utilizing curvelet reconstruction we can remove number of directional components, which are then used to evaluate the weight matrices and build the directional vectors for every pixel in various directions. Routine TV regularization produces huge relics in the resultant image, while CTV regularization term can keeps sharp edges in sixteen direction and can suppress larger number of antiquities than TV. The comparability between two small image patches can gauge by D-NLM regularization term in pixel values and the directional data of the pixel centering 
the relating patch. Obscuring, down sampling, and commotion are created in the picture obtaining procedure, and in this way the reconstruction constraint imposes a penalty on such drawbacks in the SR reconstruction process.

\subsection{Direction extraction from curvlet transform}

The Curvelet transform is a proficiently utilized for protecting edges since it has directional affectability. The curvelet coefficients of the given image $\mathrm{Y}$ can be

$$
Q=\Gamma(Y))
$$

Where $\Gamma$ (.) represent the curvelet transform and $\mathrm{Q}$ represent the set of curvlet coefficient. As $\left(Q_{j l}=\mid j=1, \ldots, J ; l=1, \ldots, L_{j}\right)$ in which $J$ and $L_{j}$ are the total number of scales and directions at the $j^{\text {th }}$ scale, respectively. Fig. II demonstrates the curvelet coefficient matrices at five sizes of a coarse-to-fine pecking order. At the primary scale, there is stand out curvelet coefficient matrix that contains smooth points of interest in the information image. From the second to fifth scales, there are 16, 32, 32, and 64 curvelet coefficient matrices, individually. The coefficient matrices are numbered from $45^{\circ}$ in clockwise request and contain more directional data at better scales.

To compute the directional components of images in 16 diverse direction can characterize as

$$
\begin{aligned}
A_{k}=\mathrm{P}\left(\mathrm{r}^{-1}\left(H_{k} \odot Q\right)\right), k=1, \ldots, 16 \\
H_{k} \odot Q=\left\{H_{k, j, l} \cdot Q_{j, l} \mid j=1, \ldots, J ; l=1, \ldots, L_{j}\right\} \\
H_{j, l}=\left\{\begin{array}{cc}
1, & \text { if }(j, l) \in Z_{k} \\
0, & \text { otherwise }
\end{array}\right.
\end{aligned}
$$

Every one of the pixels have small or large directional qualities. Prominently, the qualities in the non-edge areas are much smaller than those in the edge locales.

\subsection{Combine Total Variation (CTV)}

To change over a given LR image to its HR form, a typical decision utilized as a part of [5] is to penalize the 1-standard estimation of the image gradients, i.e., the TV estimation of the given image. The fundamental favorable position of TV regularization is that it successfully remove the ringing artifacts brought about by the additional noise. Many times minimizing the TV fails to recover sharp edges in the horizontal and vertical directions, which is for the most part because of the way that the TV regularizer ignores the direction of the edges. The DTV term [6], compensates for the 1-norm value with both direction i.e. the horizontal and vertical. This method gives very good SR results when the sharp edges are directed horizontally and vertically yet at the same time produce rough antiques along different direction, despite the fact that transform-invariant methods are utilized. An observation on natural images indicates that the sharp edges in a natural image are oriented principally in a small number of directions which induce the group sparsity property on image gradients since the non-zero values are generally grouped along the main directions. Taking into account these contemplations, in this way, we introduce a CTV term that fuses the multi-directional TV and the conventional TV of a HR estimation Y.

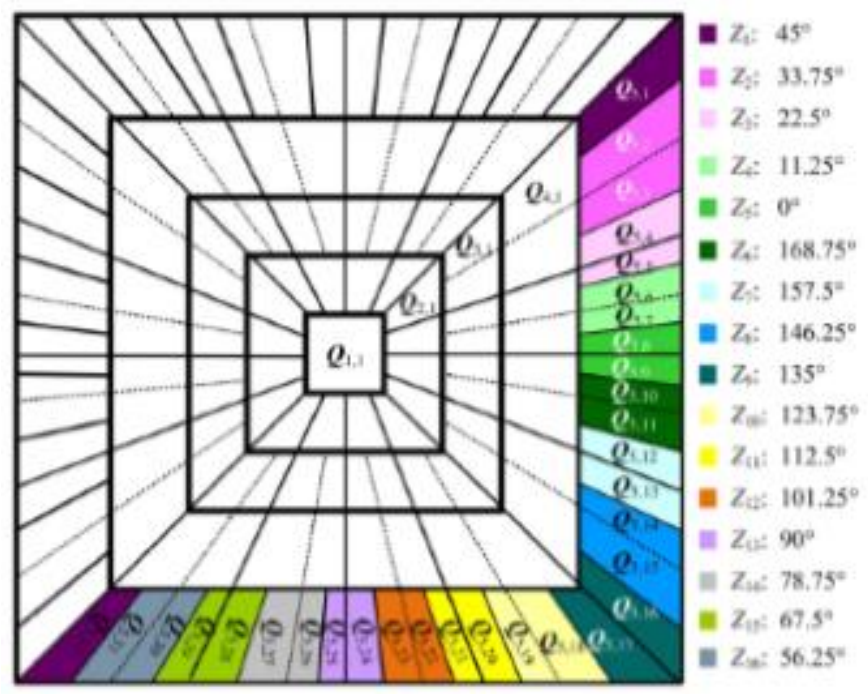

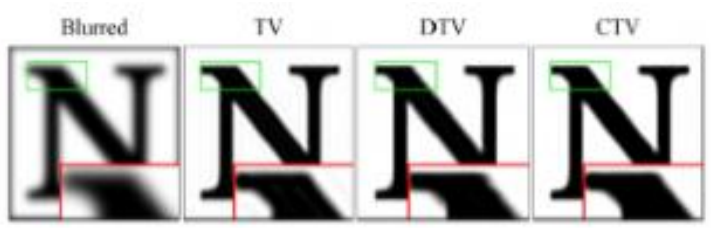

Fig. 4 Non-blind de-blurring of character ' $\mathrm{N}$ ' using TV, DTV, CTV.

Fig. 3 Curvlet coefficient matrices at different scale and partition 
Fig. 4 gives a case of TV, DTV, and CTV cost capacity minimization for the non-blind deblurring of a character "N". TV minimization produces critical artifacts along sharp edges. DTV minimization recovers the edges that are adjusted on a horizontally and vertically, be that as it may it can likewise deliver barbed ancient artifacts along the diagonal line. As opposed to TV and DTV, CTV minimization keeps the sharp edges in their main directions, and additionally smothering artifacts.

\section{Experimental Result And Analysis}

To evaluate the performance of the proposed method compared to other methods, we conduct experiments on a group of color images.
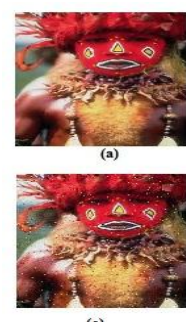

(e)

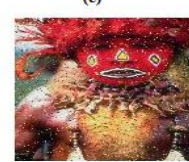

(i)

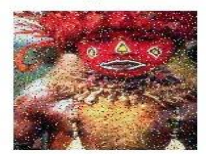

(m)
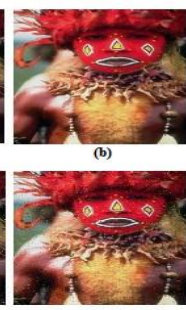

(f)

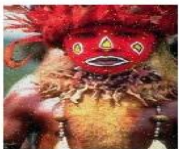

(i)

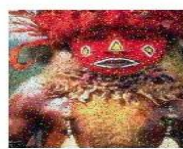

(n)
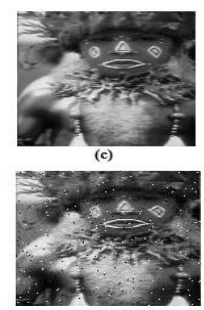

(g)

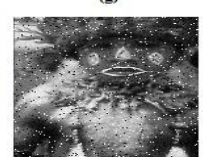

(k)

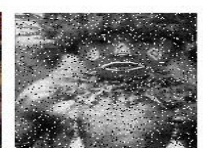

(o)
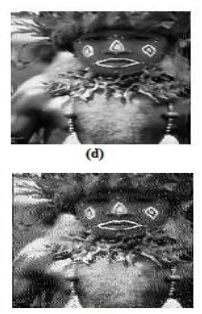

(h)

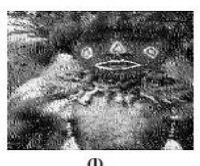

(i)

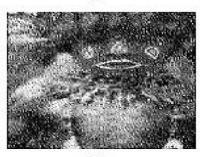

(p)

Fig. 5 Comparison of SR results for man's face image
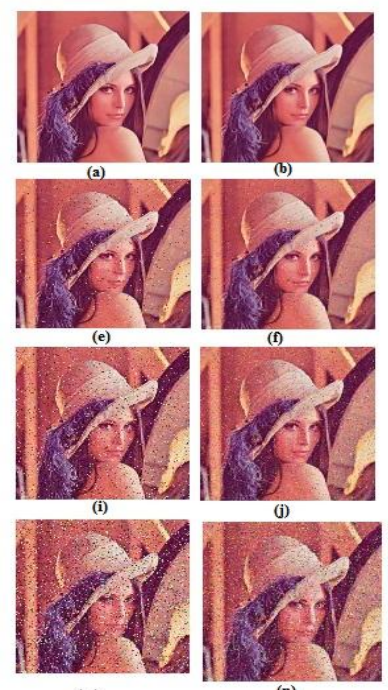

(n)
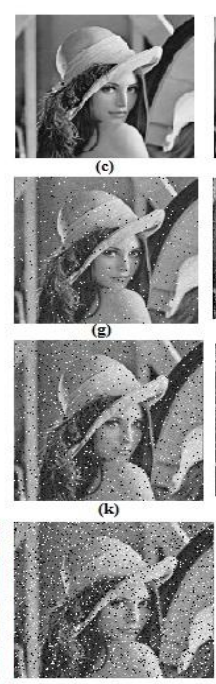

(o)
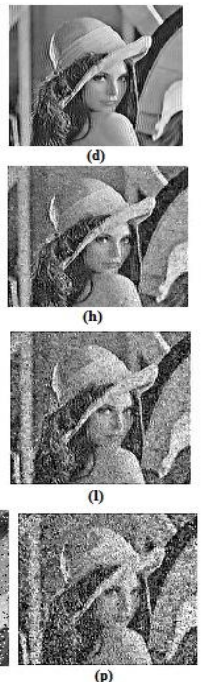

Fig. 6 Comparison of SR results for lena image
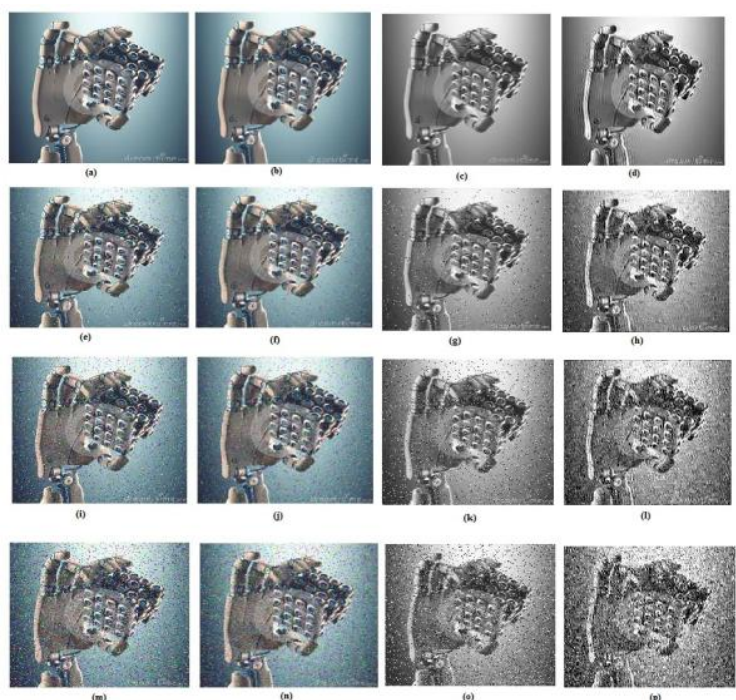

Fig. 7 Comparison of SR results for binary cube image

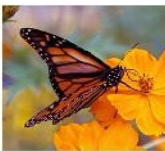

(a)
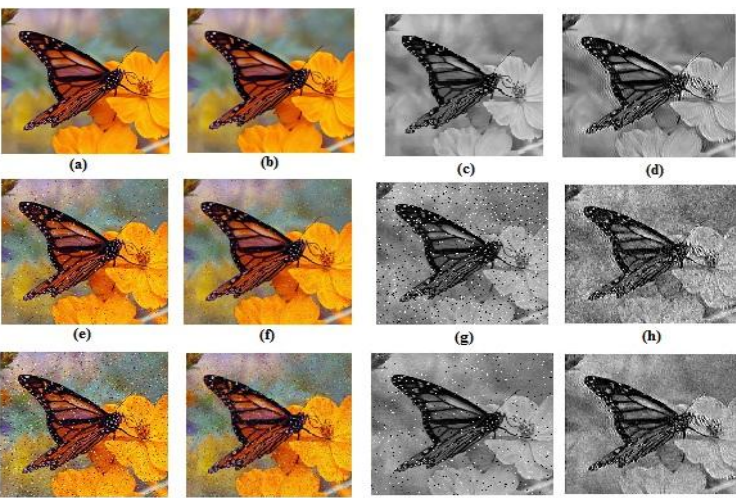

(i)

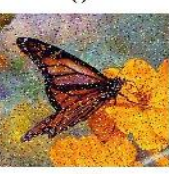

(m)
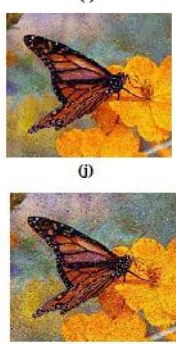

(n)

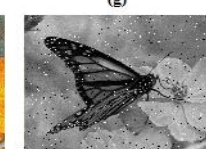

(k)
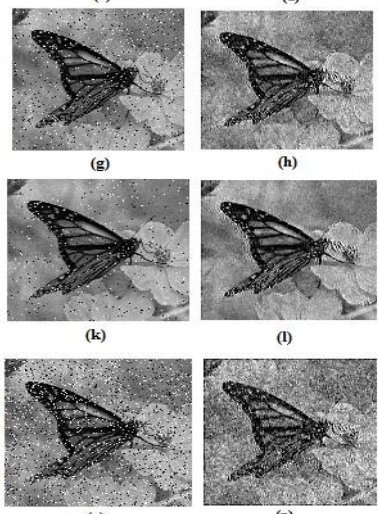

(h)

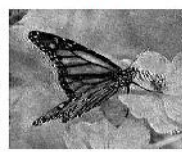

(l)

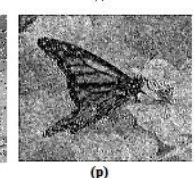

Fig. 8 Comparison of SR results for monarch butterfly image

The above all figure 5 to 8 shows the result of proposed method (a) Noiseless original image (b) Recovered image for bi-linear (c) Noiseless original image (d) Recovered image for proposed method (e) original image $(\eta=0.02)$ (f) Recovered image for bi-linear method $(\eta=0.02)$ (f) original image $(\eta=0.02)(\mathrm{g})$ Recovered image for proposed method $(\eta=0.02)(\mathrm{h})$ original image $(\eta=0.05)$ (i) Recovered image for bi-linear method $(\eta=0.05)(j)$ original image $(\eta=0.05)(k)$ Recovered image for proposed method $(\eta=0.05)(1)$ original image $(\eta=0.1)(\mathrm{m})$ Recovered image for bi-linear method $(\eta=0.1)(\mathrm{n})$ original image $(\eta=0.1)(\mathrm{o})$ Recovered image for proposed method $(\eta=0.1)$. 
We first convert the RGB channels of the color images to grey scale images and then implement the SR reconstruction. The results of proposed method are compared with conventional bi-linear method in terms of entropy, standard deviation, variance and PSNR of reconstructed images. The results of proposed method also checked for different noise values. For this noise values are 0, 0.02, 0.05, and 0.1. After adding salt and papper noise to original image the changes in recovered image is observed and also changes in parameter is checked. Quantitatively, the widely used metrics are entropy, standard deviation, variance and peak signal to noise ratio (PSNR).

Overall, bi-linear interpolation always gives the worst SR performance despite being very fast. In addition, Fig. 5 to 8 demonstrates the SR quality of the different four image using different approaches. The proposed method that incorporates CTV and D-NLM regularization terms achieves competitive results in superresolving plausible details in the textural regions, and suppressing artifacts along the sharp edges. The quantitative and qualitative results obtained by the proposed CTV-DNLM method are better, which is largely due to the fact that directional group sparsity of sharp edges can be preserved and the weights can be better estimated by using the D-NLM regularization term.

In fig. 9 and 10 for lena and Man's face Image the parameter PSNR is compared. The PSNR value for proposed method is 74.9534 as compared to Bi-linear method has 24.2827 for better than proposed method for man's face image. The man's face image give better PSNR than other test images.

Under the noisy environment, the proposed method is also superior to the other SR approaches in terms of average metric values. The proposed CTV-DNLM method is robust against noise, and is capable of preserving sharp edges and recovering textural details. This is partly because CTV and D-NLM regularization terms retain balance in suppressing noise and recovering plausible details. Due to addition of noise the PSNR value is decreases but it is still better then bi-linear method.

Table. 1 Entropy, Standard deviation, Variance, MSE and PSNR (dB) for Noiseless Images

\begin{tabular}{|c|c|c|c|c|c|c|c|c|}
\hline \multirow{2}{*}{ Parameters } & \multicolumn{2}{|c|}{ Binary Cube Image } & \multicolumn{2}{|c|}{ Man Face Image } & \multicolumn{2}{|c|}{ Lena Image } & \multicolumn{2}{c|}{ Monarch Butterfly Image } \\
\cline { 2 - 8 } & Bi-linear & $\begin{array}{c}\text { Proposed } \\
\text { Method }\end{array}$ & Bi-linear & $\begin{array}{c}\text { Proposed } \\
\text { Method }\end{array}$ & Bi-linear & $\begin{array}{c}\text { Proposed } \\
\text { Method }\end{array}$ & $\begin{array}{c}\text { Bi-linear } \\
\text { Method }\end{array}$ \\
\hline Entropy & 1.2213 & 7.6955 & 0.7440 & 7.7629 & 0.8966 & 7.7025 & 1.0631 & 7.2394 \\
\hline $\begin{array}{c}\text { Standard } \\
\text { deviation }\end{array}$ & 0.5858 & 0.2293 & 0.4118 & 0.2382 & 0.4682 & 0.2176 & 0.5190 & 0.2112 \\
\hline Variance & 0.3432 & 0.0526 & 0.1696 & 0.0568 & 0.2192 & 0.0474 & 0.2694 & 0.0446 \\
\hline MSE & 254.9994 & 0.0072 & 244.4623 & 0.0021 & 254.2751 & 0.0058 & 223.2693 & 0.0069 \\
\hline PSNR & 24.0994 & 69.5903 & 24.2827 & 74.9534 & 24.1118 & 70.5012 & 24.6765 & 69.7468 \\
\hline
\end{tabular}

Table. 2 Entropy, Standard deviation, Variance, MSE and PSNR (dB) for Images with noise $=0.05$

\begin{tabular}{|c|c|c|c|c|c|c|c|c|}
\hline \multirow[t]{2}{*}{ Parameters } & \multicolumn{2}{|c|}{ Binary Cube Image } & \multicolumn{2}{|c|}{ Man Face Image } & \multicolumn{2}{|c|}{ Lena Image } & \multicolumn{2}{|c|}{ Monarch Butterfly Image } \\
\hline & Bi-linear & $\begin{array}{l}\text { Proposed } \\
\text { Method }\end{array}$ & Bi-linear & $\begin{array}{l}\text { Proposed } \\
\text { Method }\end{array}$ & Bi-linear & $\begin{array}{l}\text { Proposed } \\
\text { Method }\end{array}$ & Bi-linear & $\begin{array}{c}\text { Proposed } \\
\text { Method }\end{array}$ \\
\hline Entropy & 1.1829 & 7.7092 & 0.7298 & 7.7121 & 0.8772 & 7.7356 & 1.0551 & 7.4291 \\
\hline $\begin{array}{l}\text { Standard } \\
\text { deviation }\end{array}$ & 0.5667 & 0.2531 & 0.4035 & 0.2570 & 0.4567 & 0.225 & 0.5156 & 0.2182 \\
\hline Variance & 0.3211 & 0.0641 & 0.1628 & 0.0661 & 0.2086 & 0.0541 & 0.2659 & 0.0476 \\
\hline MSE & 248.5431 & 0.0248 & 238.7392 & 0.0180 & 248.0849 & 0.0189 & 218.5102 & 0.0153 \\
\hline PSNR & 24.2108 & 64.2286 & 24.3856 & 65.6052 & 24.2188 & 65.4002 & 24.7701 & 66.3220 \\
\hline
\end{tabular}

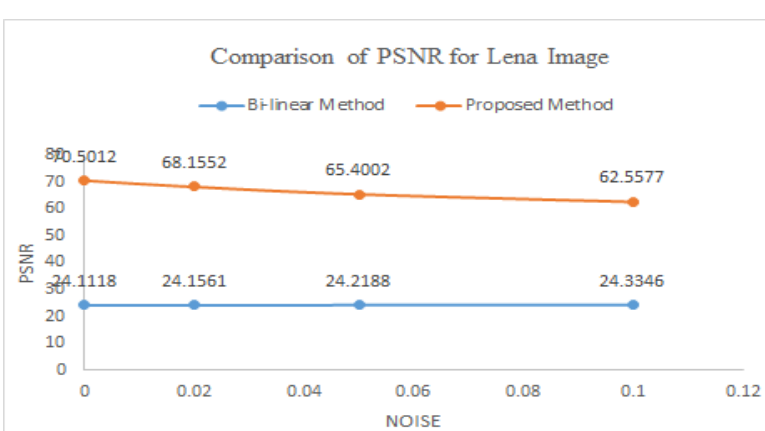

Fig. 9 Comparison of PSNR for Man’s face Image

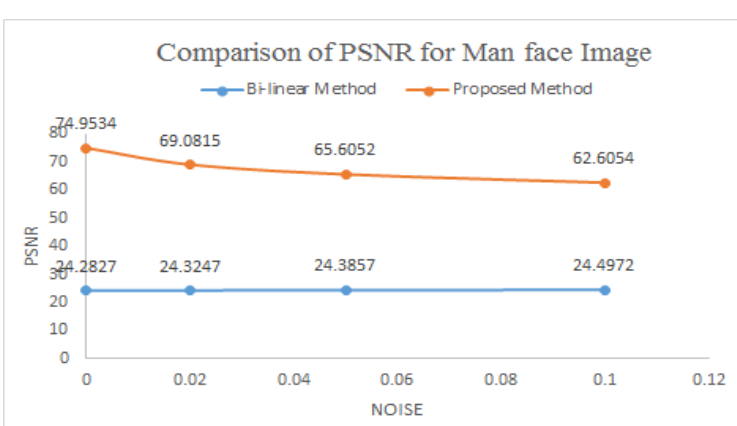

Fig. 10 Comparison of PSNR for Man's face Image

\section{Conclusion}

In SR by means of Directional features technique [1], [2] reconstruction of image taking directional group sparsity and similarity with directional features into thought. Proposed a novel reconstruction-based method for single-image super-resolution, taking directional group sparsity and self-similarity with directional features into consideration. By exploiting the directional information of the 16 different directions, the CTV 
regularizer is clearly able to preserve sharp edges. D-NLM regularization term reducing aliasing and jaggy artifacts in the final images. The proposed method incorporates the CTV and D-NLM regularization terms for solving the ill-posed problem in the SR process. The future scope of proposed method is designing a more effective feature extraction pattern for preserving sharp edges, such as deep feature and opponent features. Rising the reconstruction speed to apply in real time environments. Bad light may effect on system that can be avoided by using night vision cameras

\section{Acknowledgements}

I would like to the express my true sense and sincerest gratitude to my respected guide Dr. Mrs. S.S. Agrawal for her dynamic and valuable guidance. I am grateful to her for constant encouragement in fulfilment of paper. This work is result of combined efforts put in by my guide and me. I would also like to thank her for providing me with all necessary infrastructure and facilities to complete the paper. I extend my special thanks to PG HOD of Electronics \& Telecommunication Dr. S.K. Shaha and all staff members.

\section{Journal Papers:}

\section{References}

[1]. Xiaoyan Li, Hongjie He, Ruxin Wang, and Dacheng Tao, "Single Image Super resolution via Directional Group Sparsity and Directional Features", IEEE transaction on image processing, Vol. 24, No. 9 Sept 2015.

[2]. X. Li and M. T. Orchard, "New edge-directed interpolation," IEEE Trans. Image Process., vol. 10, no. 10, pp. 1521-1527, Oct. 2001.

[3]. X. Zhang and X. Wu, "Image interpolation by adaptive $2 D$ autoregressive modeling and soft-decision estimation," IEEE Trans. Image Ptocess, vol. 17, no. 6, pp. 887-896, Jun. 2008.

[4]. D. Tao, L. Jin, Y. Wang, and X. Li, "Person reidentification by minimum classification error-based KISS metric learning," IEEE Trans. Cybern., vol. 45, no. 2, pp. 242-252, Feb. 2015.

[5]. C. Xu, D. Tao, and C. Xu, "Multi-view intact space learning," IEEE Trans. Pattern Anal. Mach. Intell., Mar. 2015, doi: 10.1109/TPAMI.2015.2417578.

[6]. $\quad$ S. R. Becker, E. J. Candès, and M. C. Grant, "Templates for convex cone problems with applications to sparse signal recovery," Math. Program. Comput., vol. 3, no. 3, pp. 165-218, 2011.

[7]. C. Fernandez-Granda and E. J. Candès, "Super-resolution via transform- invariant group-sparse regularization," in Proc. IEEE Int. Conf. Comput. Vis, Dec. 2013, pp. 3336-3343.

[8]. D. Tao, L. Jin, Y. Wang, and X. Li, "Rank preserving discriminant analysis for human behavior recognition on wireless sensor networks," IEEE Trans. Ind. Informat., vol. 10, no. 1, pp. 813-823, Feb. 2014.

[9]. H. Takeda, S. Farsiu, and P. Milanfar, "Kernel regression for image processing and reconstruction," IEEE Trans. Image Process., vol. 16, no. 2, pp. 349-366, Feb. 2007.

[10]. Yu He, Kim-Hui Yap, Li Chen and Lap-Pui Chau, "Nonlinear least square technique for simultaneous image registration and super resolution", IEEE transactions on image processing, VOL. 16, No.11, NOV 2007 